\title{
Remarks about the Flashing Rachet
}

\author{
Jean Dolbeault* \\ Ceremade (UMR CNRS no. 7534), Université Paris IX-Dauphine, \\ Place de Lattre de Tassigny, 75775 Paris Cédex 16, France \\ E-mail: dolbeaul@ceremade.dauphine.fr \\ David Kinderlehrer ${ }^{\dagger}$ \\ Department of Mathematical Sciences, Carnegie Mellon University, \\ Pittsburgh, PA 15213, USA, \\ E-mail: davidk@andrew.cmu.edu \\ Michał Kowalczyk \\ Department of Mathematical Sciences, Kent State University, \\ Kent, $\mathrm{OH}$ 44242, USA. \\ E-mail: kowalcyk@mcs.kent.edu
}

December 9, 2003

\begin{abstract}
The flashing rachet is the simplest example of diffusion mediated transport as well as the suggested mechanism for a class of protein motors. Here we briefly explain these concepts and give an entropy based argument for existence and uniqueness of a model problem. We also examine the features of the system that lead to transport
\end{abstract}

\section{Introduction}

Diffusion mediated transport is implicated in the operation of many molecular level systems. These include some liquid crystal and lipid bilayer systems, and, especially, the motor proteins responsible for eukaryotic cellular traffic. All of these systems are extremely complex and involve subtle interactions on varying scales. In an earlier life, we were interested in the design of microstructure, typically in order to optimize the duty cycle of an actuator. In such devices, like shape memory or magnetostrictive, energy transduction is very close to equilibrium in order to minimize the energy budget - TV remotes are good examples. The chemical/mechanical

\footnotetext{
* Submitted to Proc. PASI 2003. Partially supported by ECOS-Conicyt under contract C02Exx by the CMM (UMR CNRS no. 2071),

Universidad de Chile and by the EU financed network HPRN-CT-2002-00282.

${ }^{\dagger}$ Partially supported by the National Science Foundation Grant DMS 0072194.
} 
transduction in motor proteins is, by contrast, quite distant from equilibrium. These systems function in a dynamically metastable range.

The flashing rachet is, perhaps, the simplest and most transparent example of this phenomenon. It consists of apparently competing processes: a transport, which attracts mass to specific sites, and diffusion, which spreads mass, in alternation. To give a simple and generalized formulation of this, consider this variation of the Fokker-Planck Equation:

$$
\begin{array}{ll}
\rho_{t}=\left(\sigma \rho_{x}+\psi_{x} \rho\right)_{x}, & (x, t) \in \Omega \times(0, \infty), \\
\sigma \rho_{x}+\psi_{x} \rho=0, & (x, t) \in \partial \Omega \times(0, \infty), \\
\rho(x, 0)=\rho_{0}(x), & x \in \Omega, \text { where } \\
\rho_{0} \geqq 0 \quad \text { and } \quad \int_{\Omega} \rho_{0} d x=1 &
\end{array}
$$

where $\sigma>0$, the potential $\psi=\psi(x, t)$ is a periodic function of $t$ and $\Omega=(0,1)$. Notice that if $\rho(x, 0)=\rho_{0}>0$ then $\rho(x, t)>0$ for all $t>0$. Also if $\int_{\Omega} \rho_{0}=1$ then $\int_{\Omega} \rho(x, t) d x=1$. Thus (1) in general can be thought of an evolution equation for probability density $\rho$.

The simplest example is given by

$$
\psi(x, t)= \begin{cases}\psi(x) & \text { if } 0 \leqq t \leqq T_{t r} \\ 0 & \text { if } T_{t r} \leqq t \leqq T_{d i f f}+T_{t r}=T\end{cases}
$$

which constitutes flashing between a diffusion with drift $\psi^{\prime}(x)$ and a diffusion. Of course, there is no stationary state for this type of equation, but there may be a periodic state. The problem is interesting because the periodic state is not simply some convex combination of Gibbs states, but represents a redistribution of the mass to one side of the interval $\Omega$. Here our attention will focus on the existence, uniqueness, and stability of the periodic solution by employing entropy methods. We also discuss the approximation of the periodic state in terms of a (discrete) Markov Chain using Monge-Kantorovich mass transport ideas.

\section{Existence and stability}

We outline a simple existence and stability result. Uniqueness is a consequence of the stability. Let us assume that

- $\psi$ bounded and periodic of period $T$ in $\Omega$ and $\psi \in C^{1}(\Omega \times[0, T])$.

- Then there is a unique nonnegative $T$-periodic probability density $\rho=\rho^{\sharp}$ which solves (1).

The proof is an exercise in the use of the Schauder Fixed Point Theorem employing the free energy, a convex functional, to define the convex set. Set

$$
\begin{array}{r}
E(\rho)=\sigma \int_{\Omega} \rho \log \frac{\rho}{\rho_{\psi}} d x, \quad \rho_{\psi}(x, t)=\frac{1}{\int_{\Omega} e^{-\psi(\xi, t) / \sigma} d \xi} e^{-\psi(x, t) / \sigma}, \\
\text { for } \quad \rho \in H^{1}(\Omega), \rho \geqq 0, \quad \int_{\Omega} \rho d x=1
\end{array}
$$

Assume that $\rho(x, t)$ is a solution of the Fokker-Planck Equation (1). Then

$$
\frac{d}{d t} E(\rho)=-\sigma^{2} \int_{\Omega} \rho\left|\frac{\partial}{\partial x} \log \frac{\rho}{\rho_{\psi}}\right|^{2} d x-\int_{\Omega} \frac{\partial}{\partial t} \psi \rho d x
$$

According to the log-Sobolev Inequality, for a constant $C_{\psi}$ which depends on $\psi$ and $\sigma$,

$$
\int_{\Omega} \rho \log \frac{\rho}{\rho_{\psi}} d x \leqq C_{\psi} \int_{\Omega} \rho\left|\frac{\partial}{\partial x} \log \frac{\rho}{\rho_{\psi}}\right|^{2} d x
$$

Hence, since $\psi_{t}$ is bounded and $\rho$ is a probability density for each $t$,

$$
\frac{d}{d t} E(\rho) \leqq-C_{\psi} E(\rho)+K_{\psi}
$$


and

$$
\left.E(\rho)\right|_{t=T} \leqq\left. E(\rho)\right|_{t=0} e^{-C_{\psi} T}+K_{\psi}\left(1-e^{-C_{\psi} T}\right)
$$

This means that the mapping

$$
\begin{array}{r}
T: H^{1}(\Omega) \rightarrow H^{1}(\Omega) \\
T\left(\rho_{0}\right)(x)=\rho(x, T)
\end{array}
$$

with $\rho(x, t)$ the solution of (1) with initial value $\rho_{0}$ maps the set

$$
K=\left\{\rho \in H^{1}(\Omega): E(\rho) \leqq \frac{K_{\psi}}{C_{\psi}}\right\} \cap\{\text { probability densities }\}
$$

into itself. $T$ is compact by regularity theory for parabolic equations, elementary in the $H^{1}$ case. Hence we obtain a fixed point $\rho^{\sharp}$ of $T$, which is a periodic solution of (1).

We now address the stability and uniqueness of the periodic solution. We establish a decay rate for the relative entropy of two solutions of the Fokker-Planck Equation. The familiar Csiszar-Kullback Inequality or the less familiar Talagrand Inequality may then be applied. For the moment, let $\rho_{1}$ and $\rho_{2}$ be two solutions of (1). Their relative entropy at time $t$ is

$$
E(t)=E\left(\rho_{1} \mid \rho_{2}\right)=\int_{\Omega} \rho_{1} \log \frac{\rho_{1}}{\rho_{2}} d x=\int_{\Omega} f \log f \rho_{2} d x, \quad f=\frac{\rho_{1}}{\rho_{2}} .
$$

Now compute

$$
\begin{aligned}
\frac{d}{d t} E(t) & =\frac{d}{d t} \int_{\Omega} f \log f \rho_{2} d x \\
& =\int_{\Omega}\left\{(\log f+1)\left(\frac{\partial \rho_{1}}{\partial t}-\frac{\rho_{1}}{\rho_{2}} \frac{\partial \rho_{2}}{\partial t}\right)+f \log f \frac{\partial \rho_{2}}{\partial t}\right\} d x \\
& =\int_{\Omega} \log f \frac{\partial \rho_{1}}{\partial t} d x-\int_{\Omega} f \frac{\partial \rho_{2}}{\partial t} d x
\end{aligned}
$$

We then have that

$$
\begin{aligned}
\int_{\Omega} \log f \frac{\partial \rho_{1}}{\partial t} d x & =\int_{\Omega} \log f \frac{\partial}{\partial x}\left(\sigma \frac{\partial \rho_{1}}{\partial x}+\psi_{x} \rho_{1}\right) d x \\
& =-\frac{1}{\sigma} \int_{\Omega} \frac{f_{x}}{f}\left(\frac{\rho_{1}}{\rho_{\psi}}\right) \rho_{x} d x
\end{aligned}
$$

and

$$
\begin{aligned}
-\int_{\Omega} f \frac{\partial \rho_{2}}{\partial t} d x & =-\int_{\Omega} f \frac{\partial}{\partial x}\left(\sigma \frac{\partial \rho_{2}}{\partial x}+\psi_{x} \rho_{2}\right) d x \\
& =\frac{1}{\sigma} \int_{\Omega} f_{x}\left(\frac{\rho_{2}}{\rho_{\psi}}\right) \rho_{x} d x
\end{aligned}
$$

Combining these gives that

$$
\begin{aligned}
\frac{d}{d t} E(t) & =-\frac{1}{\sigma} \int_{\Omega} \frac{f_{x}}{f}\left(\frac{\rho_{1}}{\rho_{\psi}}\right)_{x}-f\left(\frac{\rho_{2}}{\rho_{\psi}}\right) \rho_{\psi} d x \\
& =-\frac{1}{\sigma} \int_{\Omega} \frac{1}{f} f_{x}^{2} \rho_{2} d x=-\frac{1}{\sigma} \int_{\Omega}\left|\frac{\partial}{\partial x} \log \frac{\rho_{1}}{\rho_{2}}\right|^{2} \rho_{1} d x
\end{aligned}
$$




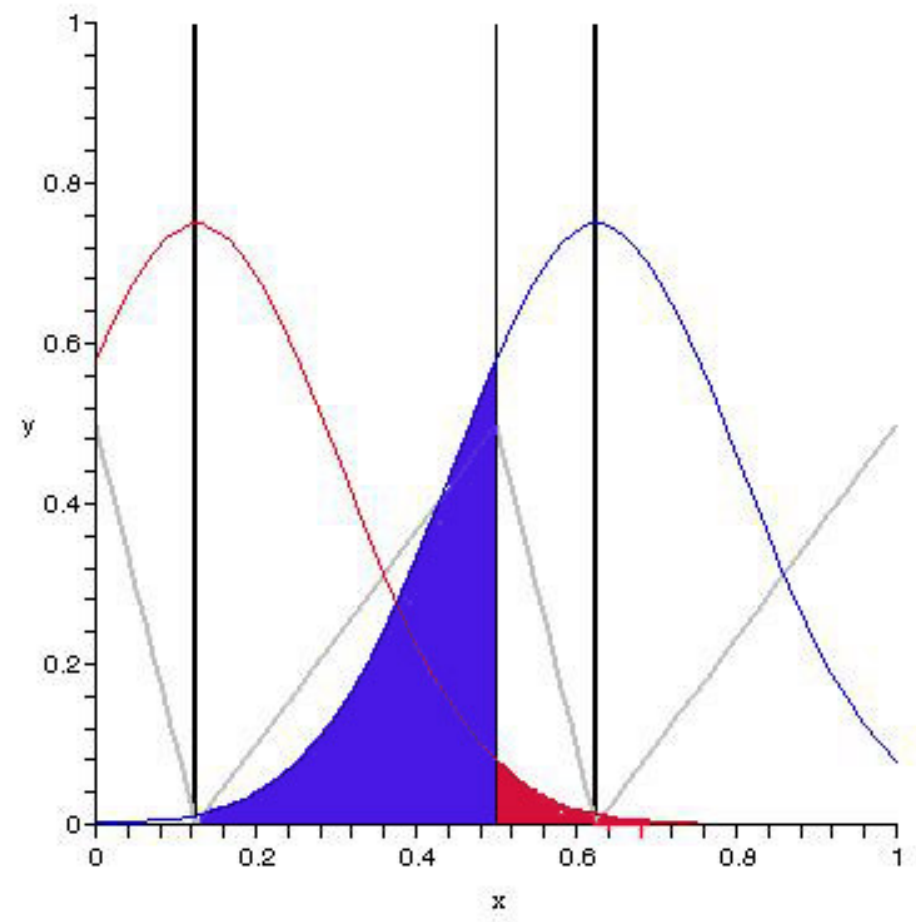

Figure 1: Two unit Dirac masses located in well basins at $x=1 / 8$ and $x=5 / 8$ diffuse. At the end of the diffusion period, more mass has moved to the left well from the right well than vice versa. In the ensuing transport step, more mass is collected to the well at $x=1 / 8$ than to $x=5 / 8$.

Now again from the log-Sobolev Inequality,

$$
\frac{d}{d t} E(t) \leqq-C E(t)
$$

where $C$ depends on $\rho_{2}$. Hence,

$$
E(t) \leqq E(0) e^{-C t / \sigma}, \quad t>0
$$

At this point it is convenient to let $\rho_{2}=\rho^{\sharp}$, the periodic solution just found. Then

$$
\int_{\Omega}\left|\rho_{1}-\rho^{\sharp}\right| d x \leqq \text { const. } e^{-C t / 2 \sigma} \quad \text { and } \quad d\left(\rho_{1}, \rho^{\sharp}\right) \leqq \text { const. } e^{-C t / 2 \sigma}
$$

by Csizsar-Kullback and Talagrand, respectively, where $d(\cdot, \cdot)$ is the Wasserstein distance. This shows both the stability and uniqueness of the periodic solution. As mentioned before, the extension to piecewise smooth in time potentials $\psi(x, t)$ like $(2)$ is achieved by concatenating the estimates, e.g., by first solving a Fokker-Planck Equation and then a diffusion equation.

\section{The mechanism of transport}

The basic mechanism of transport may be explained with a simple picture. For this, consider (2) where $\psi(x)$ is periodic in $x$ of period $1 / N$ and between maxima has an asymmetrically 
located (and unique) minimum. For example, in Figure 1, two Dirac masses located in well basins asymmetric in their period intervals diffuse for a time $T_{\text {diff }}$. Owing to the asymmetry alone, more mass moves to the left than to the right. In the ensuing transport step, more mass is collected in the left well than in the right one. When iterated, significant transport can result. This is misleading, however. It is important to know what to do with the mass when it arrives in the left-most well. In other words, boundary conditions are also extremely important. Periodic boundary conditions, for example, do not lead to transport in the flashing rachet. Our analysis of this Brownian motor renders the figure with boundary conditions rigorous by approximating the periodic solution $\rho^{\sharp}$ with a Markov chain defined on convex combinations of Dirac masses.

To arrange this, suppose that, as a typical situation, $\psi$ has maxima at $x=0,1 / N, \ldots, 1$ and minima at $x=a_{1}, \ldots, a_{N},(i-1) / N<a_{i}<i / N$. For a solution $\rho$ of $(1)$, set

$$
\begin{aligned}
\mu^{*} & =\sum_{i=1}^{N} \mu_{i}^{*} \delta_{a_{i}} \\
\mu_{i}^{*} & =\int_{I_{i}} \rho(x, t) d x, \quad I_{i}=\left[x_{i-1}, x_{i}\right], i=1, \ldots, N, 0 \leqq t \leqq T,
\end{aligned}
$$

In a moment, $\rho$ will be the periodic solution $\rho^{\sharp}$, but for the present, if

$$
\rho(x, t) \approx \mu^{*}
$$

then

$$
\begin{aligned}
\rho(x, t+T) \approx & \sum_{i=1}^{N} \mu_{i}^{*} g_{\sigma}\left(x, T, a_{i}\right) d x \\
g_{\sigma}(x, t, a)= & \text { Green's Function for the Neumann Problem } \\
& \text { with singularity a and diffusion coef ficient } \sigma .
\end{aligned}
$$

We rewrite this as

$$
\begin{aligned}
\rho(x, t+T) & \approx \mu \\
\mu & =\mu^{*} P, \quad P=\left(P_{i j}\right), \quad P_{i j}=\int_{I_{j}} g_{\sigma}\left(x, T, a_{i}\right) d x
\end{aligned}
$$

$P$ is an ergodic probability matrix. Now choose $\rho=\rho^{\sharp}$, the periodic solution and replace the * by $\sharp$ above. Then

$$
\rho^{\sharp}(x, 0) \approx \mu^{\sharp} \quad \text { and } \quad \rho^{\sharp}(x, T) \approx \mu^{\sharp} P
$$

but

$$
\rho^{\sharp}(x, 0)=\rho^{\sharp}(x, T)
$$

SO

$$
\mu^{\sharp} \approx \mu^{\sharp} P
$$

The only way these iterates of a Markov chain can be close is if $\mu^{\sharp}$, and hence $\rho^{\sharp}$, is close to the unique stationary vector $\mu^{\infty}$ of $P$. Our strategy is to show that $\mu^{\infty}$ has most of its mass on one side of $\Omega$. In summary, we find a Markov chain determined by the diffusion and the asymmetry in the system which we may attempt to exploit to characterize its transport properties. To be successful in this, the 'rachet' parameters must be appropriately tuned. We also require separate estimates for the transport and the diffusion phases. The precise distance between $\mu$ and $\rho$ will be in weak topology, namely, expressed by the Wasserstein metric $d$.

First consider the transport phase. Note that the Wasserstein distance between $\rho(x, t)$ and $\mu$ with 


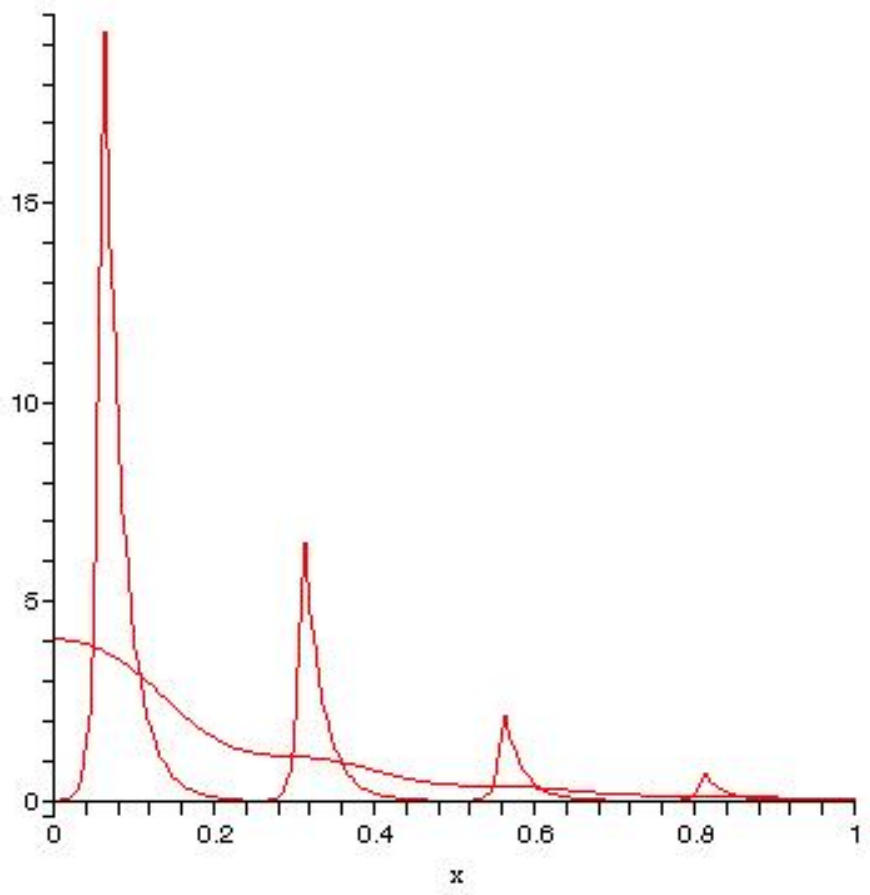

Figure 2: Snapshots of the periodic solution for a potential $\psi$ of period $1 / 4$ on $\Omega$ at the end of the transport phase, upper curve, and at the end of the diffusion phase, lower curve

$$
\mu=\sum_{i=1}^{N} \mu_{j} \delta_{a_{j}}, \quad \mu_{j}=\int_{I_{j}} \rho(x, t) d x
$$

is

$$
d(\rho, \mu)^{2}=\sum_{j=1}^{N} \int_{I_{j}}\left(x-a_{j}\right)^{2} \rho(x, t) d x
$$

To determine the rate of decay of (12), differentiate with respect to $t$ and employ the equation (1). Typically this would lead to an exponential rate of decay by a Gronwall lemma. The estimate we obtain is that

$$
\begin{aligned}
d\left(\rho^{\sharp}\left(\cdot, T_{t r}\right), \mu^{\sharp}\right) & \leqq K_{0} \omega, \text { where } \\
\omega=\omega\left(T_{t r}, T_{\text {diff }}, \sigma\right) & =\left[\frac{\log T_{t r}}{T_{t r}}+\min \left(\sqrt{\sigma} e^{\lambda T_{t r}}, 1\right)\right]
\end{aligned}
$$

$$
\text { whenever } T_{t r} \geqq T_{t r}^{*}, T_{\text {diff }} \geqq T_{\text {diff }}^{*}, 2 \pi^{2} \sigma T_{\text {diff }}-\lambda T_{\text {tr }}>\log 2
$$

Here $T_{t r}^{*}, T_{d i f f}^{*}, K_{0}$ and $\lambda$ are all constants that depend only on the potential $\psi$ and its derivatives. The $\log T_{t r} / T_{t r}$ term owes to the nonconvexity of the potential $\psi$. The second term $\min \left(\sqrt{\sigma} e^{\lambda T_{t r}}, 1\right)$ accounts for diffusion across the maxima of $\psi$, which is small when $\lambda T_{t r}$ is less than $-\log \sigma$. 
of

During diffusion, we wish to compare the two distributions $\rho(x, t)$ and $w(x, t)$, the solution

$$
\begin{aligned}
w_{t} & =\sigma w_{x x} \quad \text { in } \Omega, T_{t r}<t<T_{\text {diff }}, \\
w_{x} & =0 \text { on } \partial \Omega, T_{t r}<t<T_{\text {diff }}, \\
\left.w\right|_{t=T_{t r}} & =\sum_{i=1}^{N} \mu_{j}^{\sharp} \delta_{a_{i}}
\end{aligned}
$$

which may be accomplished in several ways, the most available of which simply employs the entropy estimate we have already proved in the previous section. This requires an estimate on $w\left(x, T_{t r}+\delta\right)$ for a small $\delta$ in order assess $E(0)$. There are some additional details to check, but in the end, we obtain a rigorous version of (10) and (11).

Finally, we address the analysis of transport as exhibited in the Markov chain $P$. How do we know that the stationary vector $p^{\infty}$ of $P$ has most of its mass in the left half of $\Omega$ when the well basins $a_{i}$ are in the left halves of their intervals $I_{i}$ ? Simulations show that this is clearly the case, cf. Figure 3 and even numerical calculation of $p^{\infty}$ are emphatic on this point. For the two-well case, i.e., $N=2$, we can verify this. In general, the slow decay of the Green's function at infinity makes estimates very difficult.

\section{Conclusions}

\section{References}

[1] A. Adjari And J. Prost, Mouvement induit par un potentiel périodique de basse symétrie: dielectrophorese pulse, C. R. Acad. Sci. Paris t. 315, Série II (1992), 1653.

[2] R.D. Astumian and M. Bier, Fluctuation driven ratchets: molecular motors, Phys. Re. Lett. 72 (1994), 1766.

[3] R.D. Astumian, Thermodynamics and kinetics of a Brownian motor, Science 276 (1997), 917-922.

[4] W. Beckner, A generalized Poincaré inequality for Gaussian measures, Proc. Amer. Math. Soc. 105 no. 2 (1989), 397-400.

[5] J.-D. Benamou And Y. BRenier, A computational fluid mechanics solution to the MongeKantorovich mass transfer problem, Numer. Math. 84 (2000), 375-393.

[6] M.J. CÁceres, J.A. Carrillo and J. Dolbeault, Nonlinear Stability in $L^{p}\left(R^{d}\right)$ for Solutions of the Vlasov-Poisson system for charged particles, to appear in SIAM J. Math. Anal.

[7] M. Chipot, D. Kinderlehrer And M. KowalczyK, A variational principle for molecular motors, Meccanica, 38, (2003)505-518

[8] W. E And P. PALfFy-Muhoray, Orientational ratchets and angular momentum balance in the Janossy effect, Mol. Cryst. Liq. Cryst. 320 (1998), 193-206.

[9] T.C. Elston And C.R. Doering, Numerical and analytical studies of nonequilibrium fluctuation-induced transport process, Jour. Stat. Physisc 83 nos. 3/4 (1996), 359-383.

[10] D. Heath, D. Kinderlehrer and M. Kowalczyk, Discrete and continuous ratchets: from coin toss to molecular motor, Discrete and continuous dynamical systems Ser. B 2 no. 2 (2002), 153-167.

[11] R. Holley, D. Stroock, Logarithmic Sobolev inequalities and stochastic Ising models, J. Stat. Phys. 46 nos. 5/6 (1987), 1159-1194.

[12] J. Howard, Mechanics of Motor Proteins and the Cytoskeleton, Sinauer Associates, Inc., 2001. 
[13] R. Jordan, D. Kinderlehrer and F. Otto, The variational formulation of the FokkerPlanck equation, SIAM J. Math. Anal. Vol. 29 no. 1 (1998), 1-17.

[14] F. Jülicher And J. Prost, Cooperative molecular motors, Phys. Rev. Lett. 75 (1995), 2618.

[15] D. Kinderlehrer and M. Kowalczyk, Diffusion-mediated transport and the flashing ratchet, Arch. Rat. Mech. Anal. 161 (2002), 149-179.

[16] D. Kinderlehrer and N. Walkington, Approximation of parabolic equations based upon Wasserstein's variational principle, Math. Model. Numer. Anal. (M2AN) 33 no. 4 (1999), 837-852.

[17] T. Kosa, W. E and P. Palffy-Muhoray, Brownian motors in the photoalignment of liquid crystals, Int. J. Eng. Science 38 (2000), 1077-1084.

[18] Y. OKada AND N. Hirokawa, A processive single-headed motor: kinesin superfamily protein KIF1A, Science Vol. 283, 19 February 1999.

[19] Y. Okada And N. Hirokawa, Mechanism of the single headed processivity: diffusional anchoring between the K-loop of kinesin and the $C$ terminus of tubulin, Proc. Nat. Acad. Sciences 7 no. 2 (2000), 640-645.

[20] Отто, F., Dynamics of labyrinthine pattern formation: a mean field theory, Arch. Rat. Mech. Anal. 141 (1998), 63-103

[21] Отто, F., The geometry of dissipative evolution equations: the porous medium equation, Comm. PDE 26 (2001), 101-174

[22] Otto, F. And C. Villani Generalization of an inequality by Talagrand and links with the logarithmic Sobolev Inequality, J. Funct. Anal. 173, (2000), 361-400

[23] A. Van OudenaARden AND S. Boxer, Brownian ratchets: molecular separation in lipid bilayers supported on patterned arrays, Science 285 (1999), 1046-1048.

[24] A. Parmeggiani, F. Jülicher, A. Adjari and J. Prost, Energy transduction of isothermal ratchets: generic aspects and specific examples close and far from equilibrium, Phys. Rev. E, 60 no. 2 (1999), 2127-2140.

[25] C.S. Peskin, G.B. Ermentrout And G.F. Oster, The correlation ratchet: a novel mechanism for generating directed motion by ATP hydrolysis, in Cell Mechanics and Cellular Engineering (V.C Mow et.al eds.), Springer, New York, 1995.

[26] P. Reimann, Brownian motors: noisy transport far from equilibrium, Phys. Rep. 361 nos. 2-4 (2002), 57-265.

[27] R.D. Vale And R.A. Milligan, The way things move: looking under the hood of motor proteins, Science 288, 7 April 2000, 88-95.

[28] C. Villani Topics in optimal transportation, AMS (2003) 